\section{Postbiotics - when simplification fails to clarify}

\author{
José Eleazar Aguilar-Toalá, Stefania Arioli, Pradip Behare (i), \\ Clara Belzer, Roberto Berni Canani(D), Jean-Marc Chatel, Enza D’Auria(D), \\ Mônica Queiroz de Freitas, Eran Elinav (1), Erick Almeida Esmerino, \\ Hugo S. García, Adriano Gomes da Cruz, Aarón F. González-Córdova, \\ Simone Guglielmetti@i), Jonas de Toledo Guimarães (1),

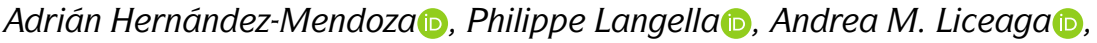 \\ Marciane Magnani, Rebeca Martin, Mohammad Tamrin Mohamad Lal, \\ Diego Mora, Mehran Moradi(D), Lorenzo Morelli, Fabio Mosca, \\ Filomena Nazzaro, Tatiana Colombo Pimentel(D), Chao Ran, \\ Chaminda Senaka Ranadheera (1), Maria Rescigno, Azucena Salas (1), \\ Anderson S. Sant'Ana (D), Katia Sivieri(D), Harry Sokol(D, Valentina Taverniti, \\ Belinda Vallejo-Cordoba, Jaroslav Zelenka and Zhigang Zhou
}

We appreciate the intent by Salminen et al. to clarify and reorganize the nomenclature regarding the use of inactivated bacteria and their products as health-promoting factors (Salminen, S. et al. The International Scientific Association of Probiotics and Prebiotics (ISAPP) consensus statement on the definition and scope of postbiotics. Nat. Rev. Gastroenterol. Hepatol. 18, 649-667 (2021)) $)^{1}$. Nonetheless, we found several major caveats in the consensus statement ( $\mathrm{BOX} 1$ ) that might generate ambiguity.

Salminen et al. ${ }^{1}$ redefined the term postbiotic, including also inactivated microorganisms, stating that "the term 'postbiotics' $\ldots$ is inconsistently used and lacks a clear definition". However, the definition of postbiotic was explicitly enunciated in 2013 as "any factor resulting from the metabolic activity of a probiotic or any released molecule capable of conferring beneficial effects to the host in a direct or indirect way" ${ }^{2}$, in agreement with other proposed definitions ${ }^{3,4}$. Despite being true that the term is increasingly found in the scientific literature and on commercial products ${ }^{1}$, it is most largely used in accordance with the original definition ${ }^{2}$, and not according to the new meaning proposed by the ISAPP ${ }^{1}$. When we searched PubMed using the term 'postbiotics', we found 220 pertinent publications (113 review and 107 research articles; search performed 7 Jun 2021). In only $14 \%$ of these papers (including work by some of the consensus authors ${ }^{5,6}$ ) did postbiotic encompass inactivated bacteria.

We also felt that there were potential issues in reviewing the scientific literature concerning inactivated microorganisms. Salminen et al. reported that scientific publications adopted either the wording 'non-viable probiotics', 'heat-killed probiotics', 'tyndallized probiotics', 'postbiotics' or 'paraprobiotic'. However, the first three are periphrases that only partially refer to inactivated cells (some just refer to an inactivation method or to the viability feature) and are, therefore, different from the omni-comprehensive term paraprobiotic, coined with the purpose to encompass all these descriptions of inactivated cells ${ }^{7}$. According to PubMed, 56 scientific publications in the past 10 years (22 review and 36 research articles; search performed 7 Jun 2021) used 'paraprobiotic' to explicitly indicate inactivated and/or dead bacteria (that is, in accordance with the original definition of this term ${ }^{7}$ ). No other terms have been more extensively adopted so far to indicate the use of inactivated microorganisms with health-promoting properties.

According to its original definition, postbiotics are well-defined mixtures of (or single) molecules with demonstrated benefit for the host (which would encompass health benefits) and do not include inactivated cells. The use of inactivated and/or dead microorganisms implies health benefits originating from a multitude of molecular factors that might interact synergistically or additively. This aspect applies also to conventional probiotics (how to indicate the precise molecules or structures determining the whole spectrum of health benefits for probiotics such as Lacticaseibacillus rhamnosus GG or Lacticaseibacillus paracasei Shirota). For this reason, we believe that a scientific need exists to distinguish well-defined molecular factors of microbial origin with health-promoting properties (original concept of postbiotic)

\section{Box 1 | List of caveats of the ISAPP consensus statement on postbiotics}

- Disagreement with the proposed new definition of postbiotic: the ISAPP consensus statement ${ }^{1}$ proposes a common term to simultaneously indicate inactivated microorganisms and their specific products or components, which, in our opinion, generates confusion as well-defined molecular factors cannot be distinguished from complex (undefined) matrices derived from microbial cells.

- Review of the available scientific literature: the nomenclature adopted by the scientific literature in the past 10 years concerning inactivated microorganisms and their molecules and/or factors has been, in our opinion, mostly overlooked or misinterpreted. In particular, we believe the meaning most commonly attributed to the word 'postbiotic' in scientific literature has been ignored.

- Stability of products including inactivated microorganisms: the ISAPP's document stated that "products with a long shelf life can be readily achieved for inanimate microorganisms" also in "geographical regions that do not have reliable cold chains or whose ambient temperature causes problems for storage of live microorganisms", concluding that products containing inactivated microorganisms can "likely be extremely stable for several years at room temperature". This claim is potentially unrealistic as the shelf life of any food, supplement or drug is influenced by its constituents (inorganic, organic or enzymatic) that inevitably participate to processes profoundly affecting stability (such as enzymatic modifications, Maillard reactions or oxidations), especially when temperature is not controlled, unsuitable packaging is implemented and/or improper storage conditions are adopted.

- Valid markers of efficacy in products containing inactivated microorganisms are needed. It is of pivotal importance that a valid parameter is selected to test a preparation including inactivated microbial cells to effectively prove the preservation of the efficacy (that is, its health-promoting property) during the shelf life. This point is particularly challenging and should have been discussed further.

- Use of the word "inanimate": the word "inanimate" is not generally used in the field of microbiology with the meaning proposed by the ISAPP's consensus statement. The proposed explanation that 'inanimate' should be a better wording to indicate non-viable or dead microorganisms because 'inactivated' could erroneously be intended as "an inert material" ${ }^{1}$ does not seem to have a scientific need.

ISAPP, International Scientific Association of Probiotics and Prebiotics. 
from the use of complex matrices derived from microbial cells, for which the precise molecular factors supporting the health benefits are not comprehensively known (concept of paraprobiotic). The scientific literature of the past 10 years supports this distinction.

In science, heterogeneous groups are required only when unique definitions are not applicable. Here, definitions are not only possible, but useful and already available. There is no need for fading edges when we can mark them, ensuring a clear distinction.

There is a reply to this letter by Salminen, $\mathrm{S}$. et al. Reply to: Postbiotics - when simplification fails to clarify. Nat. Rev. Gastroenterol. Hepatol. https://doi.org/10.1038/s41575-02100522-5 (2021).

José Eleazar Aguilar-Toalán , Stefania Arioli Pradip Behare (iD ${ }^{3}$, Clara Belzer ${ }^{4}$ Roberto Berni Canani iD ${ }^{5}$, Jean-Marc Chatel ${ }^{6}$, Enza D'Auria iD 7 ${ }^{7}$, Mônica Queiroz de Freitas ${ }^{8}$, Eran Elinav (iD ${ }^{9,10}$, Erick Almeida Esmerino ${ }^{8}$, Hugo S. García ${ }^{11}$, Adriano Gomes da Cruz ${ }^{12}$, Aarón F. González-Córdova ${ }^{13}$, Simone Guglielmetti (iD) ${ }^{2 \times}$ Jonas de Toledo Guimarães iD ${ }^{8}$ Adrián Hernández-Mendoza (iD ${ }^{13}$, Philippe Langella (iD ${ }^{6}$, Andrea M. Liceaga iD ${ }^{14}$, Marciane Magnani ${ }^{15}$ Rebeca Martin ${ }^{6}$, Mohammad Tamrin Mohamad Lal' ${ }^{16}$, Diego Mora ${ }^{2}$, Mehran Moradi iD ${ }^{17}$, Lorenzo Morelli' ${ }^{18}$, Fabio Mosca ${ }^{19,20}$, Filomena Nazzaro ${ }^{21}$, Tatiana Colombo Pimentel (iD ${ }^{22}$, Chao Ran ${ }^{23}$, Chaminda Senaka Ranadheera (iD ${ }^{24}$ Maria Rescigno ${ }^{25,26 凶}$, Azucena Salas (iD ${ }^{27}$, Anderson S. Sant'Ana iD ${ }^{28}$, Katia Sivieri iD ${ }^{29}$,

Harry Sokol iD ${ }^{6,30,31}$, Valentina Taverniti ${ }^{凶}$ Belinda Vallejo-Cordoba ${ }^{13}$, Jaroslav Zelenka iD ${ }^{32}$ and Zhigang Zhou ${ }^{33}$

Laboratory of Transformation and Emerging Technologies in Foods, National Autonomous University of Mexico, FES-Cuautitlan, Mexico.

${ }^{2}$ Division of Food Microbiology and Bioprocesses, Department of Food, Environmental and Nutritional Sciences, University of Milan, Milan, Italy.

${ }^{3}$ Dairy Microbiology Division, ICAR-National Dairy Research Institute Karnal, Karnal, Haryana, India.

${ }^{4}$ Laboratory of Microbiology, Wageningen University, Wageningen, Netherlands.

${ }^{5}$ Department of Translational Medical Science, ImmunoNutritionLab at CEINGE Advanced Biotechnologies Research Center, European Laboratory for the Investigation of Food Induced Diseases,

Task Force for Microbiome Studies - University of Naples "Federico II", Naples, Italy.

${ }^{6}$ Université Paris-Saclay, INRAE, AgroParisTech,

MICALIS UMR1319, Jouy-en-Josas, France. ${ }^{7}$ Allergy Unit, Department of Pediatrics, Vittore Buzzi Children's Hospital, University of Milan, Milan, Italy.

${ }^{8}$ Faculty of Veterinary, Federal University Fluminense, Niterói, Brazil

9Immunology department, Weizmann Institute of Science, Rehovot, Israel.
${ }^{10}$ Microbiome and Cancer Division, DKFZ, Heidelberg, Germany.

${ }^{11}$ Unidad de Investigación y Desarrollo de Alimentos, TecMN-Instituto Tecnológico de Veracruz, Veracruz, Mexico.

${ }^{12}$ Department of Food, Federal Institute of Education, Science and Technology of Rio de Janeiro (IFRJ), Rio de Janeiro, Brazil.

${ }^{13}$ Laboratorio de Química y Biotecnología de Productos Lácteos, Centro de Investigación en Alimentación y Desarrollo A.C., Hermosillo, Mexico.

${ }^{14}$ Department of Food Science, Protein Chemistry \& Bioactive Peptides Lab, Purdue University, West Lafayette, IN, USA

${ }^{15}$ Laboratory of Microbial Processes in Foods, Department of Food Engineering, Federal University of Paraíba, João Pessoa, Brazil.

${ }^{16}$ Borneo Marine Research Institute, Universiti Malaysia Sabah, Sabah, Malaysia.

${ }^{17}$ Department of Food Hygiene and Quality Control, Faculty of Veterinary Medicine, Urmia University, Urmia, Iran.

${ }^{18}$ Department for Sustainable Food Processes, Università Cattolica del Sacro Cuore, Piacenza, Italy. ${ }^{19}$ Neonatal Intensive Care Unit, Fondazione IRCCS Ca

Granda Ospedale Maggiore Policlinico (IRCCS), Milan, Italy.

${ }^{20}$ Department of Clinical Sciences and Community Health, University of Milan, Milan, Italy. ${ }^{21}$ Institute of Food Science - Italian National Research Council, Avellino, Italy.

${ }^{22} \mathrm{Federal}$ Institute of Paraná, Campus Paranavaí, Paraná, Brazil.

${ }^{23}$ Key Laboratory for Feed Biotechnology of the Ministry of Agriculture and Rural Affairs, Institute of

Feed Research, Chinese Academy of Agricultural Sciences, Beijing, China.

${ }^{24}$ School of Agriculture \& Food, Faculty of Veterinary \& Agricultural Sciences, The University of Melbourne, Melbourne, Victoria, Australia.

${ }^{25}$ IRCCS Humanitas Research Hospital, Milan, Italy. ${ }^{26}$ Department of Biomedical Sciences, Humanitas University, Pieve Emanuele, Italy.

${ }^{27}$ IDIBAPS, CIBEREHD, Barcelona, Spain

${ }^{28}$ Department of Food Science and Nutrition, Faculty of Food Engineering, University of Campinas, Campinas, Brazil.

${ }^{29}$ Department of Biological Sciences, Faculty of Pharmaceutical Sciences, State University of São Paulo (UNESP), Araraquara, Brazil.

${ }^{30}$ Sorbonne Université, INSERM, Centre de Recherche Saint-Antoine, CRSA, AP-HP, Saint Antoine Hospital,

Gastroenterology Department, Paris, France.

${ }^{31}$ Paris Center for Microbiome Medicine (PaCeMM) FHU, Paris, France.

${ }^{32}$ Department of Biochemistry and Microbiology, University of Chemistry and Technology Prague Prague, Czechia.

${ }^{33}$ China-Norway Joint Lab on Fish Gastrointestinal Microbiota, Institute of Feed Research, Chinese Academy of Agricultural Sciences, Beijing, China.

凶e-mail: simone.guglielmetti@unimi.it; lorenzo.morelli@unicatt.it; maria.rescigno@ hunimed.eu; valentina.taverniti@unimi.it https://doi.org/10.1038/s41575-021-00521-6
1. Salminen, S. et al. The International Scientific Association of Probiotics and Prebiotics (ISAPP) consensus statement on the definition and scope of postbiotics. Nat. Rev. Gastroenterol. Hepatol. 18 649-667 (2021)

2. Tsilingiri, K. \& Rescigno, M. Postbiotics: what else? Benef. Microbes 4, 101-107 (2013).

3. Aguilar-Toalá, J. E. et al. Postbiotics: an evolving term within the functional foods field. Trends Food Sci. Technol. 75, 105-114 (2018).

4. Patel, R. M. \& Denning, P. W. Therapeutic use of prebiotics, probiotics, and postbiotics to prevent necrotizing enterocolitis: what is the current evidence? Clin. Perinatol. 40, 11-25 (2013).

5. Collado, M. C., Vinderola, G. \& Salminen, S. Postbiotics: facts and open questions. A position paper on the need for a consensus definition. Benef. Microbes 10, 711-719 (2019).

6. Mantziari, A., Salminen, S., Szajewska, H. \& Malagon-Rojas, J. N. Postbiotics against pathogens commonly involved in pediatric infectious diseases. Microorganisms 8, 1510 (2020).

7. Taverniti, V. \& Guglielmetti, S. The immunomodulatory properties of probiotic microorganisms beyond their viability (ghost probiotics: proposal of paraprobiotic concept) Genes Nutr. 6, 261-274 (2011).

\section{Competing interests}

S.A. has research projects sponsored by Sofar, Sacco, Sanofi and Actial Farmaceutica. P.B. has been receiving grants from Ministry of Food Processing Industries for health-promoting exopolysaccharides derived from lactic and probiotic bacteria. C.B. has led research projects sponsored by several industry partners such as Danone-Nutrica, FreislandCampina and Sensus. R.B.C. had the following relevant financial relationships with the following manufacturers: Ch. Hansen (research grant, speaker), iHealth (research grant), Humana (research grant), Kraft-Heinz (research grant, speaker), Mead Johnson Nutrition (research grant, speaker), Nestlè (research grant, speaker), Novalac (research grant), Nutricia (research grant, speaker) and Sofar (research grant) as part of publically funded research projects with the support of the Italian Ministry of Health. E.E. is the founding scientist of DayTwo and BiomX. None of the activities of these entities is related to the topics presented in this commentary. S.G. has led research projects sponsored by several industry partners such as Sofar, 7 Inv, Sacco, Metis Healthcare, Giellepi and Perfetti-Van Melle. S.G. is a consultant for Sofar and Perfetti Van Melle. S.G. receives royalties from the sale of probiotic bacterial strains. P.L. has led research projects with several agro-food (Danone, General Mills, Dupond and Lallemand), food supplements (Pilèje), biotech (Ysopia) and pharmaceutical (Biocodex and Merck) companies and is a co-founder of Exeliom bioscience. M. Magnani has participated in research projects sponsored by industrial partners such as Farmoquímica and Danone Nutricia, the latter not for study of probiotics. D.M. is in the Scientific Advisory Board of Actial Farmaceutica. L.M. is consultant at Bayer, Ferrero, Sanofi and DSM and shareholder in Advanced Analytical Technologies. FN participated in a research project funded by Eurolactis (Luxemburg). M.R. has received funding for research collaborations by Kraft-Heinz; Gelesis; and AlfaSigma. She is in the advisory board of MillBo and Gelesis. She is founder and CSO of Postbiotica. K.S. has projects sponsored by industrial partners such as Nestle and Danone Nutricia, the latter not for the study of probiotics or prebiotics. H.S. received unrestricted study grants from Danone, Biocodex and Enterome; board membership, consultancy, or lecture fees from Carenity, Abbvie, Astellas, Danone, Ferring Mayoly Spindler, M.S.D. Novartis, Roche, Tillots, Enterome, Maat, BiomX, Biose, Novartis and Takeda; and is a co-founder of Exeliom bioscience. V.T. receives royalties from the sale of viable and inactivated probiotic bacterial strains. M. Moradi declares that he has no task or primary function other than the research and education in his university/country. The other authors declare no competing interests. 